\title{
Methadone - An old analgesic with new tricks
}

\author{
DE Moulin MD
}

$\mathrm{M}_{\mathrm{a}}^{\mathrm{e}}$ ethadone is a unique opioid analgesic that has been available in generic form for decades. It is available as two stereoisomers that impart different mechanisms of action. The l-isomer of methadone is a potent opioid agonist and the $\mathrm{d}$-isomer has $\mathrm{N}$-methyl-D-aspartate antagonist properties. $\mathrm{N}$-methyl-D-aspartate antagonism may play an important role in the management of neuropathic pain and may reverse morphine tolerance and hyperalgesia (1). In addition, methadone blocks the reuptake of monoamines, similar to the action of tricyclic antidepressants (2). Methadone has other attractive pharmacological properties, including high oral bioavailability, long duration of action and lack of active metabolites. The challenging aspects of methadone's use as an analgesic are tremendous interindividual variation in pharmacokinetics and variable potency based on previous opioid exposure.

In this issue of Pain Research $\mathbb{8}$ Management, Lynch (pages 133-144) provides an elegant review of methadone's analgesic properties, and also provides clear practical guidelines for the use of methadone in the management of chronic pain (3). This review is timely, because methadone is being increasingly recognized as a valuable second-line opioid analgesic for chronic noncancer pain, especially chronic neuropathic pain (4-6). In contrast to methadone's apparent new role in the treatment of chronic noncancer pain, there is extensive literature on the use of methadone for cancer pain, and Lynch appropriately takes advantage of this literature (7-9) to formulate her guidelines. The cancer pain literature provides a very useful model for opioid-switching to methadone from more conventional opioid analgesics such as morphine, and this common strategy for managing cancer pain is being used increasingly in the management of noncancer pain. Lynch also wisely advocates methadone dosing every $8 \mathrm{~h}$ by the oral route of administration, because most patients obtain adequate analgesia with this schedule (10). Although a significant number of patients respond to extended dosing intervals, time-contingent dosing every $8 \mathrm{~h}$ makes dose titration much more user-friendly than pain-contingent dosing, given methadone's propensity to interindividual variation in pharmacokinetics and variable potency based on previous opioid exposure. There is, however, one important distinction in dose titration between chronic cancer pain and noncancer pain. Cancer pain is often acuteon-chronic pain, and there may be an urgency to implement rapid dose titration despite the variable pharmacokinetics and potency. In contrast, noncancer pain rarely presents with this kind of urgency, and we have the luxury of gradual dose titration. Because the elimination half-life of methadone can be $24 \mathrm{~h}$ or longer (11), dose increases every five to seven days to achieve steady-state at each dose level minimizes the risk of serious adverse events such as respiratory depression.
The role of methadone as an analgesic has been stigmatized by its longstanding use in methadone maintenance programs for opioid addiction. This is responsible, in Canada, for the requirement to obtain an exemption to prescribe methadone under federal authority, with the actual recommendation for methadone use delegated to the provincial medical licensing body. This is a significant and unfortunate regulatory barrier to the use of methadone for analgesic purposes. We now have guidelines for the use of methadone for the treatment of chronic noncancer pain sponsored by the Colleges of Physicians and Surgeons of Ontario (12) and Nova Scotia (3). Hopefully, these guidelines, endorsed by regulatory authorities, will lead to the elimination of the requirement for an exemption to prescribe methadone for analgesic purposes. Methadone has undergone a renaissance because of some unique properties that may make it especially useful for the management of neuropathic pain, but it is still just another opioid analgesic that can be prescribed safely with appropriate guidelines.

\section{REFERENCES}

1. Davis AM, Inturrisi CE. d-Methadone blocks morphine tolerance and $\mathrm{N}$-methyl-D-aspartate-induced hyperalgesia. J Pharmacol Exp Ther 1999;289:1048-53.

2. Codd EE, Shank RP, Schupsky JJ, Raffa RB. Serotonin and norepinephrine uptake inhibiting activity of centrally acting analgesics: Structural determinants and role in antinociception. J Pharmacol Exp Ther 1995;274:1263-70.

3. Lynch ME. A review of the use of methadone for the treatment of chronic non-cancer pain. Pain Res Manage 2005;10:143-54.

4. Gagnon B, Almahrezi A, Schreier G. Methadone in the treatment of neuropathic pain. Pain Res Manage 2003;8:149-54.

5. Morley JS, Bridson J, Nash TP, Miles JB, White S, Makin MK. Low-dose methadone has an analgesic effect in neuropathic pain: A double-blind randomized controlled crossover trial. Palliat Med 2003; 17:576-87.

6. Moulin DE, Palma D, Watling C, Schulz V. Methadone in the management of intractable neuropathic non-cancer pain. Can J Neurol Sci 2005;32:340-3.

7. Ripamonti C, Groff L, Brunelli C, Polastri D, Stavrakis A, De Conno F. Switching from morphine to oral methadone in treating cancer pain: what is the equianalgesic dose ratio? J Clin Oncol 1998;16:3216-21.

8. Bruera E, Pereira J, Watanabe S, Belzile M, Kuehn N, Hanson J. Opioid rotation in patients with cancer pain. A retrospective comparison of dose ratios between methadone, hydromorphone, and morphine. Cancer 1996;78:852-7.

9. Mercadante S, Casuccio A, Fulfaro F, et al. Switching from morphine to methadone to improve analgesia and tolerability in cancer patients: A prospective study. J Clin Oncol 2001;19:2898-904.

10. Bruera E, Sweeney C. Methadone use in cancer patients with pain: A review. J Palliat Med 2002;5:127-38.

11. Davis M, Walsh D. Methadone for the relief of cancer pain: A review of pharmacokinetics, pharmacodynamics, drug interactions and protocols of administration. Support Care Cancer 2001;9:73-83.

12. Cunningham G, Bodley S, Chaiet A, et al. Methadone for Pain Guidelines: Facilitated by the Ontario College of Physicians and Surgeons. 2004. <www.cpso.on.ca/Publications/methpain.htm> (Version current at August 12, 2005) 


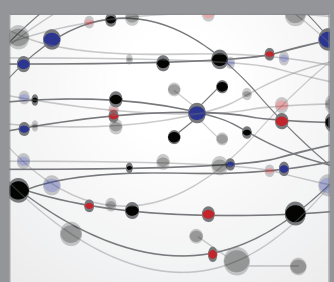

The Scientific World Journal
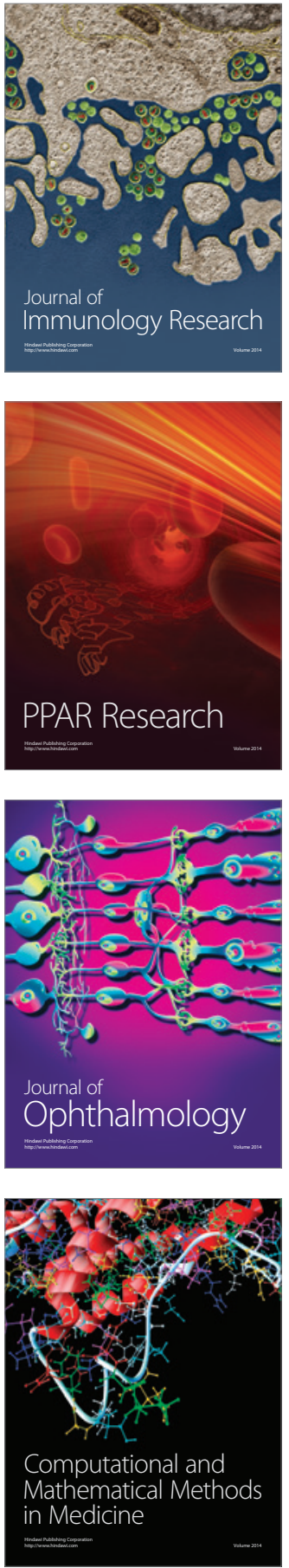

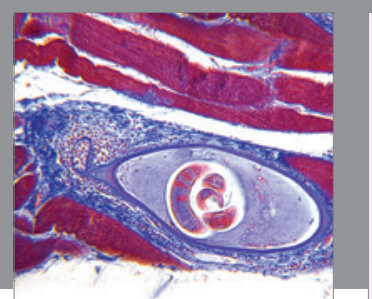

Gastroenterology Research and Practice

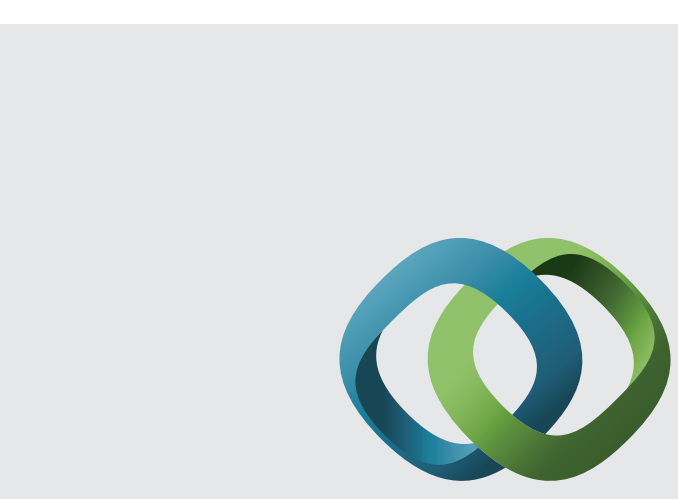

\section{Hindawi}

Submit your manuscripts at

http://www.hindawi.com
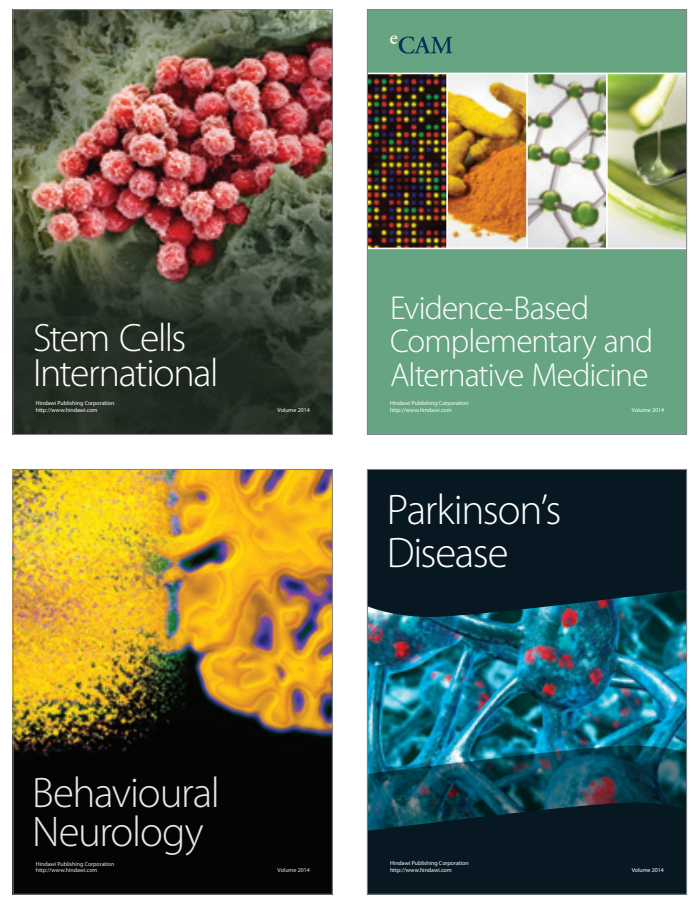
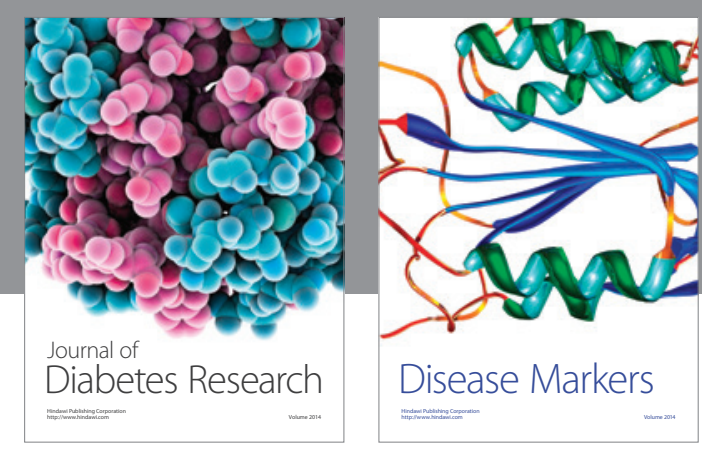

Disease Markers
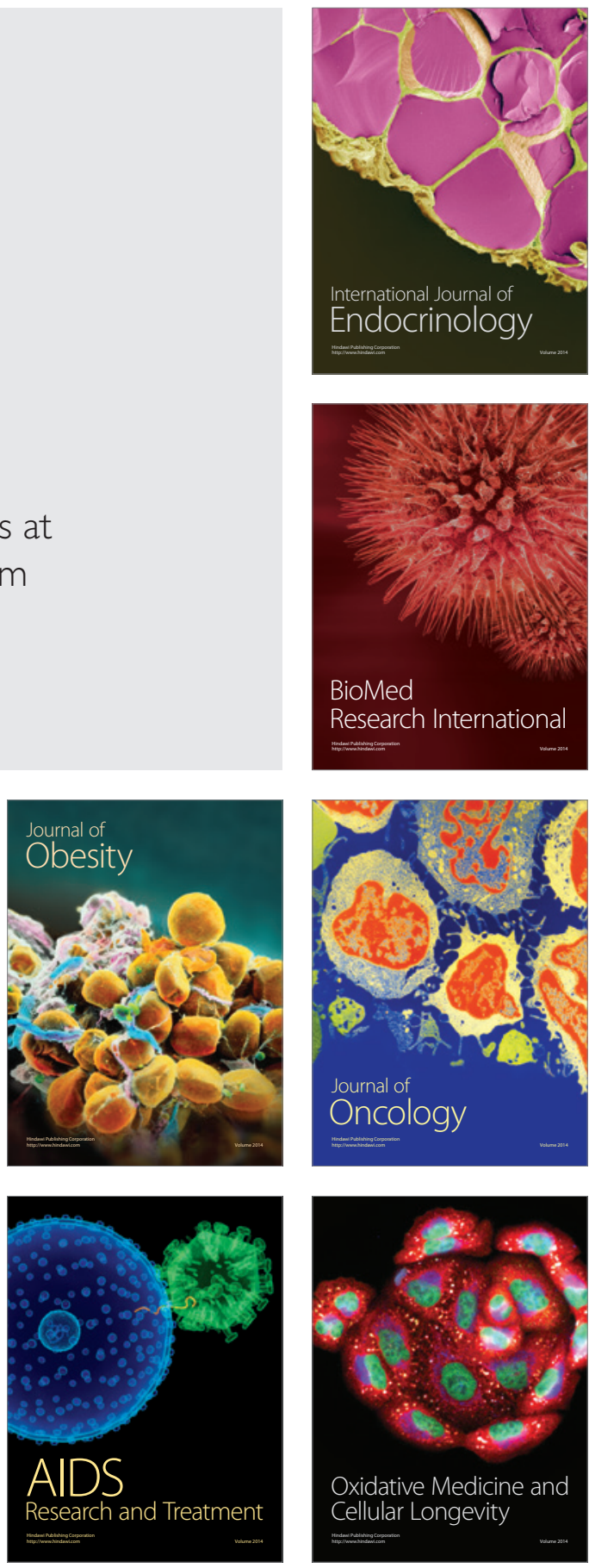